\title{
AUTOMATED MANUFACTURE OF 3D REINFORCED AEROSPACE COMPOSITE STRUCTURES
}

\author{
GIUSEPPE DELL'ANNO*, IVANA K. PARTRIDGE \\ Composites Centre, School of Applied Sciences, Cranfield University, \\ Cranfield, United Kingdom ${ }^{\dagger}$ \\ giuseppe.dellanno@cranfield.ac.uk \\ www.cranfield.ac.uk \\ DENIS D. R. CARTIÉ, ALEXANDRE HAMLYN \\ Coriolis Composites \\ Quéven, France \\ denis.cartie@coriolis-composites.com \\ www.coriolis-composites.com \\ EDMON CHEHURA, STEPHEN W. JAMES, RALPH P. TATAM \\ Engineering Photonics Group, School of Engineering, Cranfield University, \\ Cranfield, United Kingdom \\ e.chehura@cranfield.ac.uk \\ www.cranfield.ac.uk
}

\begin{abstract}
Purpose- This study is part of the FP7 project ADVITAC and focuses on exploring an innovative combination of cutting edge technologies to be implemented within automated processes for composite parts manufacturing. The objective is the design of a production route for components with tailored fibre orientation and ply lay-up, with improved damage tolerance thanks to through-the-thickness reinforcement and integrated health monitoring systems based on optical fibres technology.
\end{abstract}

Design/Methodology/Approach- The proposed technologies are described in detail and their compatibility and potential for integration are discussed.

A set up for on-line monitoring of infusion and curing processes of carbon/epoxy laminates preformed by dry fibre placement technology is proposed, and a preliminary study of their mechanical performance is presented. The possibility of reinforcing through-the-thickness preforms manufactured with dry slit tapes automatically laid-up and consolidated by laser heating is investigated.

Findings- Improved knowledge of interaction/compatibility between the discussed technologies and scope for application.

Research limitations/implications (if applicable)- The paper reports the technical potential and practical feasibility of the proposed integrated production process. Limited quantitative evaluations on the materials performance are provided. The analysis of the technologies involved represents the early outcome of the ongoing ADVITAC project.

Practical implications (if applicable)- This study contributes to the identification of a new generation of composite architecture which allows production cost and weight savings while retaining the level of quality suitable for demanding structural applications, with particular relevance to the aerospace field.

Originality/value- This paper investigates for the first time the practical possibility of designing a single automated process involving dry fibre placement, tufting and optical fibre sensor monitoring for the production of complex composite components.

\footnotetext{
${ }^{*}$ Corresponding author
} 


\section{Introduction}

This study is part of the FP7 project ADVITAC ${ }^{1}$, which aims at designing and developing a novel composite tail cone. Innovative aspects concerning the manufacture of the component involve the use of automated dry fibre placement, its combination with through-the-thickness reinforcement via tufting and the online monitoring of the process via optical fibre sensors. To date, there is no evidence in the literature of an integrated use of these technologies in a single automated manufacturing process.

Robotic dry fibre placement is based on the use of bindered tows that allow process outcome quality comparable to that of the traditional prepreg/autoclave route in terms of fibre alignment and fibre volume fraction. The high quality achieved is accompanied by the capability to process using low cost routes such as infusion. This can then be combined with another robotic technology to enhance greatly the delamination cracking resistance of the structure via tufting. Application of through-the-thickness reinforcement is being explored in this project, since localised reinforcement is seen as necessary to achieve the required damage tolerance for aerospace applications and truly lightweight integrated composite structures. Tufting causes minimal disturbance of fibre orientations in the in-plane directions, thus preserving the high mechanical performance resulting from dry fibre placement. The improvements in structural performance and process cost implied by these developments are accompanied by advances in the process quality guided by on-line monitoring. Fibre optic sensors are utilised to monitor the progress of critical stages of the manufacturing process, namely resin infusion and cure, as well as the strain distribution throughout the part during its service life.

This paper is articulated in sections describing each of these technologies in detail and focussing on their potential to be implemented within a single fully automated and integrated manufacturing process for the production of complex composite structures for real-life aeronautical applications. It is believed that the proposed production route may contribute to:

- improve the durability of the manufactured parts by tailoring the fibre architecture to the predicted load patterns by automated dry fibre placement,

- preserve their structural integrity and improve their damage tolerance by reducing the effects of composite delamination by tufting,

- monitor their structural integrity throughout their lifetime by strategic placement of optical fibre sensors.

The integration of these technologies appears particularly beneficial when considering their potential to complement each other and contribute synergistically to preserve the integrity of the composite structure. A tailored fibre placement allows full exploitation of the fibre load-bearing capabilities, thus minimising the risk of failure. On the other hand, while through-the-thickness reinforcements have, in general, no effect on crack initiation, they can significantly improve the resistance of the laminate to propagation of an existing crack (Koh et al., 2011). At the same time, sensors placed strategically within the part can monitor the strain/stress fields during its lifetime, thereby providing feedback on the integrity of the structure while in use.

\section{Robotic Dry Fibre Placement}

Automated tape laying (ATL) and automated dry fibre placement (ADFP) are well established technologies for the production of a large variety of composites parts in the aeronautic industry. Their advantage towards fabric or large tape manual lay-up consists mainly of the ability to place consistently the fibre at the right place and with the correct orientation, in order to achieve the mechanical characteristics demanded to primary loadbearing structures. Automation also promotes consistency in the quality of the produced parts, often obtained in a fraction of the time, compared to manual methods. The possibility of producing larger components, such as aircraft fuselages, is another advantage of these methods together with the ability of achieving near net-shape performs, sensibly reducing material wastage and, hence, costs. All these aspects make of ATL and ADFP the 
ideal candidate for the production of helicopter panels and blades, tail cones, components for business jets, short and long range civil aircraft, military aircraft, engine nacelles and fan blades.

Fibre placement equipment available on the market today is mostly based on machine tool concepts (Sloan, 2008; Grant, 2000; Izco et al., 2006). These have been developed on a milling or drilling machine basis and optimized for particular parts or applications, resulting in a lack of flexibility in terms of tool movements and dimensions. Investment and running costs are high due to the complex heavy industrial machinery involved, which often yield low volume production, with high energy consumption and costly dedicated maintenance. The need for flexibility and modularity has led to the development on new systems mostly based on poly-articulated robots, which are able to be adapted or re-programmed to different processes and different applications, with reduced risk of dependence on a single program or single contract. These units are able to handle a variety of raw materials and to provide high production rates while working on complex geometries and challenging structures.

Nowadays, standard off-the-shelf poly-articulated robots are widely developed and have been produced for many years for the requirements of the automotive industry. They have reached a very high level of reliability and appear to be ideally suited for use within an ADFP system able to satisfy all the requirements listed above. These poly-articulated robots have payloads ranging from $6 \mathrm{~kg}$ to $1 \mathrm{ton}$, and are capable of accuracies from $0.15 \mathrm{~mm}$ to $0.5 \mathrm{~mm}$. They can be combined with linear axis up to $60 \mathrm{~m}$ length and spindle axis up to $40 \mathrm{~m}$. Up to 6 robots can collaborate together increasing the potential for modularity of such systems. The mean average costs range from $€ 50,000$ to $€ 120,000$ per robot cell, the mean time between failures being 40,000 hours.

One of the main advantages of utilizing robots for ADFP processes is in their ability to have a large range of movement, which, nevertheless, may also represent its biggest challenge. The key aspect, in fact, arising from the use of robots for fibre placement is designing a suitable fibre feed mechanism to the head. Existing methods are based on feeding or guiding the fibres from the creel using a pulley system, which, apart from being excessively complex, poses risks of damage to the fibre and does not solve the problem of re-spooling the bobbin when the arm retracts from its operating position. A possible solution might consist of mounting the spools (or the creel itself) directly onto the deposition head. However, this option usually leads to either limiting severely the amount of fibres the head can handle or to using robots with very high payloads, hence reducing speed and accuracy of the process.

The setup investigated within this work relies on a standard off-the-shelf robot and on an innovative fibre placement system, patented by Coriolis Composites (France). The system, described below, consists of a placement or deposition head, a creel (spool cabinet) and a set of fibre-feeding pipes (figure 1).

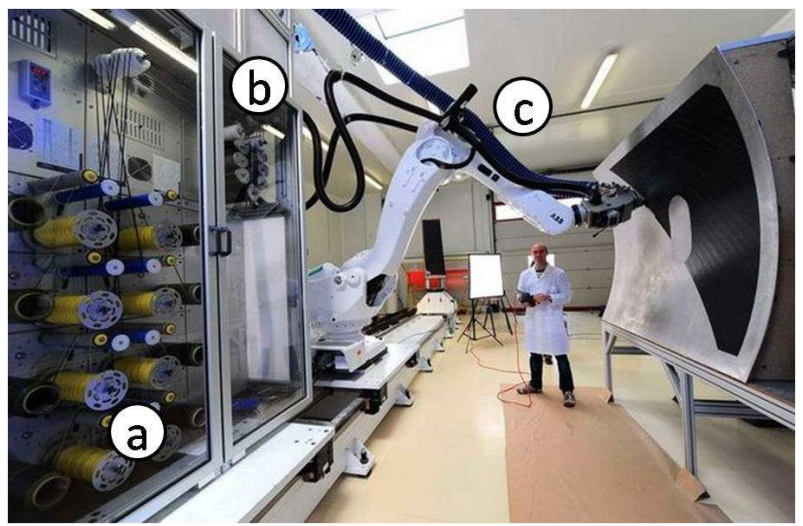

Fig. 1. Architecture of the Coriolis Composites ADFP system: (a) creel, (b) tape delivering system, and (c) temperature controlled tape feeding pipes. 
Although such an ADFP system is capable of handling the three main types of materials currently used in fibre placement processes ${ }^{2}$, its adoption within the present project focuses only on the use of stabilised dry tows. This is because such a material allows the use of liquid resin injection routes for component manufacturing, typically less expensive than prepregging and autoclave based systems (Ridgard, 2009).

Strictly speaking, the term 'dry fibre' is not completely accurate; in reality, in order to be processed by an ADFP machine, the fibres need to be stabilized, or 'bindered'. Several methods to do this are commercially available, usually based on the use of a thermoplastic or thermoset binder (typically in the form of a powder) coating the dry tows. The binding agent content is in the order of a few percent. The material is not tacky at room temperature, therefore, in order to lay-up the plies successfully, the binding agent has to be activated. This activation consists of applying, via the robot controlled deposition head, heat and pressure onto the tow in order to melt the thermoplastic particles. Depending on materials, the activation temperature ranges from $80{ }^{\circ} \mathrm{C}$ to 180 ${ }^{\circ} \mathrm{C}$. Current technologies use hot air torches for heat input and the head compaction roller for applying pressure. The use of hot air torches gives satisfactory results but the lay-up speeds are relatively low (250 $\mathrm{mm} / \mathrm{s}$ to 300 $\mathrm{mm} / \mathrm{s})$.

The following paragraphs describe in detail the ADFP system considered within the present study.

\subsection{The robot}

The ADFP system analysed in this paper uses the 6 motion axes of the poly-articulated robot (supplied either by $\mathrm{ABB}^{3}$ or by $\mathrm{KUKA}^{4}$ ) plus two external ones: a mould guiding unit and a robot positioning axis, therefore, the complete cell typically has 8 degrees of freedom. The robot, as well as the whole cell, complies with the aeronautic specifications in terms of fibre placement and cutting accuracy, as well as of repeatability and reliability of the process.

It is important to highlight that the tufting system described in Section 3 also adopts KUKA robots for handling the tufting tool. This implies that, in principle, one single robotic arm could be used for laying the dry fibres first and subsequently to tuft the preform, simply by swapping tools in between the two steps. Multifunctional robots able to select different tools for different tasks are already widely used in the automotive industry and are relatively easy to export to different environments as long as a suitable robot/tool interfaces are designed.

\subsection{The fibre feeding system}

In order to solve the complex fibre guiding problem while maintaining a large range of movements to the robot, the ADFP unit described here adopts a system based on the use of flexible pipes. Each fibre is guided through its own pipe or tube, with a rectangular, hand-made section of non abrasive plastic material. The fibre is protected in a close shell while it moves along the robotic arm and the temperature within this enclosed environment is carefully controlled and adjusted according to the various needs.

The first fibre is fed through using a dummy leader. To date, the pipes have proved their reliability over three years on the first production model. Analysis of the inside walls showed no wear and tear and no gunging up. This result was expected given that the fibres are under low tension and kept at a relatively low temperature. The rigidity of the tubes and their rectangular section reduce the risk of breakage or twisting, even during complex movements of the robot.

\subsection{The deposition head}

The head was designed for use on smaller robots so it is relatively compact and light (figure 2). It comprises three units that can be separately taken apart: heating unit, steering unit, and mobile unit, including the cutting restarting systems, the guidance plates and the electro-pneumatic triggers. The head is $550 \mathrm{~mm}$ long, it is $75 \mathrm{~mm}$ to $150 \mathrm{~mm}$ wide and weighs from $40 \mathrm{~kg}$ to $70 \mathrm{~kg}$, depending on the number of tows. Due to the close location of the cutting knives from the nip point, the minimal fibre length that the system is able to lay on even surfaces is 
$80 \mathrm{~mm}$. The compacting force can be adjusted between $2 \mathrm{~N} / \mathrm{mm}$ and $10 \mathrm{~N} / \mathrm{mm}$ to enable placements on different surfaces, such as honeycombs, relatively 'soft' inserts and more complex profiles.

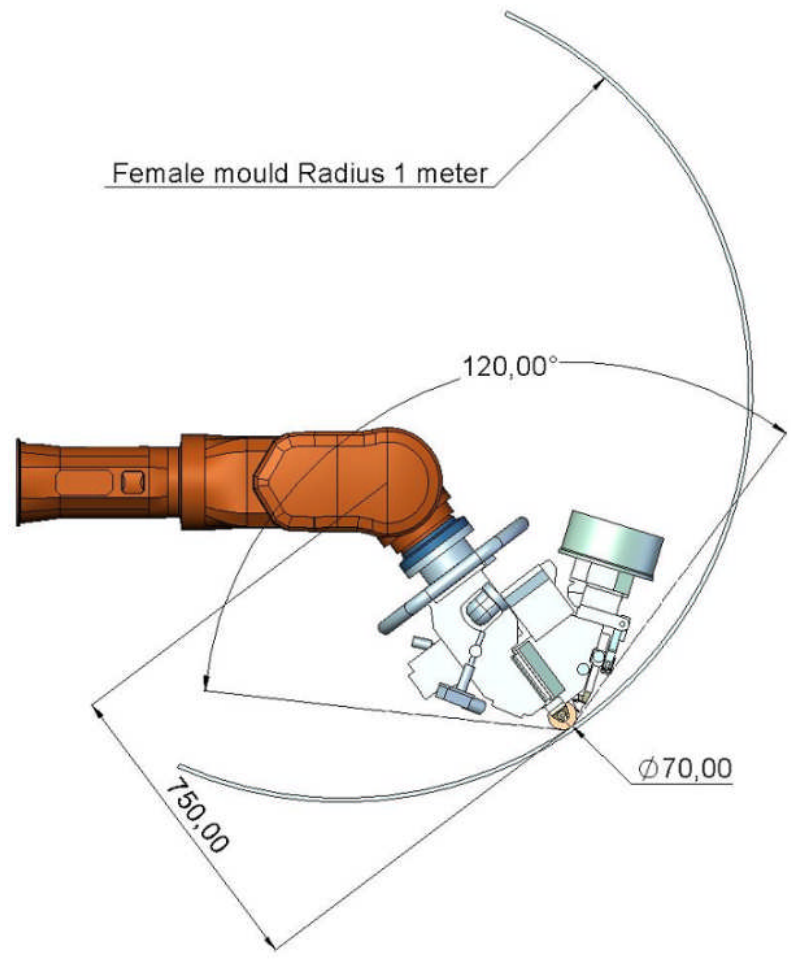

Fig. 2. Schematic of ADFP deposition head.

The standard heating system is an infrared lamp, adjustable between $30{ }^{\circ} \mathrm{C}$ to $200{ }^{\circ} \mathrm{C}$, heating the area immediately ahead of placement. However, the modular design of the head allows fast change of the heating unit to hot air torches if higher temperatures need to be reached (up to $700{ }^{\circ} \mathrm{C}$ ) or faster ramps (hence shorter deposition times) are required. The use of high power lasers promises to provide more control and better quality of the deposition compared to the other heating systems, together with high heating rate. Heating lasers were trialled on the ADFP unit within the course of this study and lay-up speeds in the order of $1 \mathrm{~m} / \mathrm{s}$ were reached while retaining a very good quality of the preform. Tuftability trials were conducted on laser-compacted preforms as well as mechanical tests on impregnated panels; detailed account of the results is given in Sections 3.1 and 2.6 , respectively.

\subsection{The creel}

The spools of the creel run freely, their motion occurs through a patented pre-delivery device, called Multiwinch $^{\circledR}$. Its role is to feed the head on command while reducing the tensile stress to negligible values within the whole range of placement speeds. Each bobbin is set within a single unit with individual electrical and pneumatic connectors. The unit contains spooling, unspooling interleaf (only for prepreg tapes) and axis braking devices and is equipped with stress and rotation sensors. The creel stops the robot head in case the fibre breaks, a bobbins empties, there is a faulty start on a new fibre placement, or if a damaged reel gives an overload signal by spooling off. 


\subsection{The software}

A software package dedicated to fibre placement has been created to take into account all the constraints of composite manufacturing and robotics. CADFibre ${ }^{\mathrm{TM}}$ and CATFibre ${ }^{\mathrm{TM}}$ (the version integrated into CATIA DELMIA) have been designed using a modular architecture, each module fulfilling a dedicated task.

The Design Module includes the numerical tools to import the CAD surface and the geometry of the manufacturing tools and jigs, allowing construction of the ply sequences. The tools necessary for the analysis of the quality of a laminate draped on complex surfaces are included. The fibre covering simulation tools include analysis of fibre angle deviation and steering analysis. If necessary, a ply (defined by its contour and a reference curve or ply direction) can be cut automatically into sectors in order to fulfil maximum angle deviations.

The Composites Manufacturing Module allows generation of tapes, i.e. bands of several fibres. Pre-manufacturing checks can be performed thanks to various analysis tools including fibre compaction, roller crush, and tool path viewing. Special algorithms to optimize head tilt to prevent collisions, draping fibres around corners and optimization of the roller contact with complex surfaces (such as ramps of honeycombs) are also selectable.

The Robotic Simulation Module includes all the tools necessary for the creation of a machine programme from the constructed lay-up. These include: definition of the link path strategy to optimize cycle time, collision detection with the various elements existing in the work cell, post processing in order to create robot programmes in the machine native language, and finally the creation of a statistic report giving simulated cycle times and details of material use.

The robot is calibrated in the manufacturer's factory and that calibration correction factors are integrated into its control device. The trajectory repeatability is $+/-0.5 \mathrm{~mm}$ at max load and max speed. The repeatability in position is $+/-0.1 \mathrm{~mm}$ at max load. Once the head is integrated as the robot tool, the absolute accuracy in position is within $+/-0.5 \mathrm{~mm}$ at max load. In order to prevent any overlap while laying up, a gap of $0.5 \mathrm{~mm}$ is pre-programmed between tapes in order to maintain the $-0 /+0.5 \mathrm{~mm}$ requirement.

\subsection{Mechanical performance of ADFP laminates}

Once the dry perform has been laid-up (figure 3), it can be injected with resin using classical RTM or infusion processes or, if required, reinforced locally by tufting, as described in Section 3.

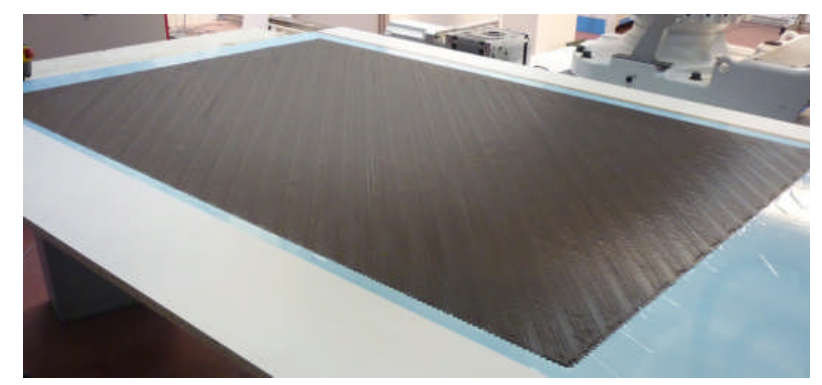

Fig. 3. Dry fibre preform made by automatic dry fibre placement.

For the purpose of this study, preforms made with two types of dry unidirectional carbon fibre tapes (Hexcel AS7 J12K V800 or Porcher 12K mod. HR EPR5311, both $6.35 \mathrm{~mm}$ wide) were impregnated with three grades of epoxy resin (HexFlow RTM6, Cycom 890RTM or Prism EP2400) by vacuum infusion technique, and cured according to the resin manufacturer technical datasheets. Cure completeness was verified by Differential Scanning Calorimetry. The laminates detailed in table I were manufactured; fibre volume fraction was evaluated theoretically on the basis of simple geometric considerations, taking into account the tape areal weight, fibre density, number of plies and the measured thickness of the cured panels. Fibre content is within acceptability range and close to the values typically obtained with prepreg laminates. 
Table I. Details of ADFP carbon/epoxy laminates manufactured.

\begin{tabular}{lcccc}
\hline Materials combination & Tape areal weight & No. of plies & Thickness & Fibre volume fraction \\
\hline Hexcel tape/HexFlow RTM6 & $252 \mathrm{~g} / \mathrm{m}^{2}$ & 8 & $2.1-2.2 \mathrm{~mm}$ & $51 \%-54 \%$ \\
Hexcel tape/Prism EP2400 & & & $2.1-2.2 \mathrm{~mm}$ & $51 \%-54 \%$ \\
Porcher tape/Cycom 890RTM & $133 \mathrm{~g} / \mathrm{m}^{2}$ & 16 & $1.9-2.0 \mathrm{~mm}$ & $59 \%-63 \%$ \\
\hline
\end{tabular}

Fibres were laid-up either in unidirectional arrangement (for tensile tests) or in biaxial $+/-45^{\circ}$ (for in-plane shear test). Tensile tests were conducted according to ASTM D3039, at a loading rate of $2 \mathrm{~mm} / \mathrm{min}$, both in longitudinal and transverse direction. In-plane shear tests followed BS EN ISO 14129, using a loading rate of 2 $\mathrm{mm} / \mathrm{min}$, with the ultimate shear strength being evaluated at a shear strain of $5 \%$. The results are summarised in table II.

Table II. Summary of results of mechanical tests on ADFP carbon/epoxy laminates. Errors represent one standard deviation.

\begin{tabular}{llccc}
\hline Test & Property & $\begin{array}{c}\text { Hexcel tape } \\
\text { HexFlow RTM6 }\end{array}$ & $\begin{array}{c}\text { Hexcel tape } \\
\text { Prism EP2400 }\end{array}$ & $\begin{array}{c}\text { Porcher tape } \\
\text { Cycom 890RTM }\end{array}$ \\
\hline Tensile $0^{\circ}$ & Modulus [GPa] & $142 \pm 3$ & $143 \pm 7$ & $135 \pm 5$ \\
& Strength [MPa] & $2160 \pm 100$ & $2200 \pm 190$ & $2220 \pm 150$ \\
Tensile 90 & Ultimate strain [\%] & $1.44 \pm 0.13$ & $1.45 \pm 0.05$ & $1.53 \pm 0.08$ \\
& Modulus [GPa] & $7.2 \pm 0.1$ & $7.8 \pm 0.3$ & $7.8 \pm 0.5$ \\
& Strength [MPa] & $33.3 \pm 2.4$ & $47.2 \pm 7.9$ & $62.1 \pm 4.1$ \\
In-plane shear & Ultimate strain [\%] & $0.46 \pm 0.03$ & $0.41 \pm 0.28$ & $0.83 \pm 0.06$ \\
& Modulus [GPa] & $3.8 \pm 0.3$ & $4.1 \pm 0.5$ & $4.2 \pm 0.3$ \\
& Strength [MPa] & $63 \pm 1.3$ & $61.6 \pm 2.5$ & $68.3 \pm 0.5$ \\
\hline
\end{tabular}

The longitudinal tensile results, as expected, are governed by the fibre: the two Hexcel based laminates have identical elastic response, whereas the modulus of the Porcher laminate appears slightly lower, suggesting this fibre might be more compliant than the other system. The results of the transverse tensile test show that the Hexcel tape/Hexflow RTM6 system has a slightly lower modulus when compared to the Porcher tape/Cycom 890RTM and to the Hexcel tape/Prism EP24000, which have an identical elastic response instead. The values recorded for strength and ultimate strain are lower than expected, especially for the Hexcel tape based systems. Nevertheless, approximately $80 \%$ of the transverse tensile test coupons failed in proximity of the tabs which might have prompted an early failure. The relatively low scatter of the in-plane shear test results demonstrates high reproducibility. The elastic response is very similar for the different materials, whereas the Porcher system is slightly stronger than both the Hexcel ones. However, this could be due to the smaller number of layers in the latter ( 8 instead of 16) which reduces significantly the interaction between the fibres with different orientation, hence reducing the measured strength.

\section{Through-the-thickness Reinforcement by Tufting}

The lack of fibres running along the $\mathrm{Z}$ direction (i.e. in the direction perpendicular to the main composite plane) in standard composite laminates leaves the matrix resin to bear the out-of-plane loads, making the composite susceptible to failure by delamination (Cartié et al., 2006). Several techniques involving the alteration of the fibre arrangement and aiming to achieve a three-dimensional fibre architecture have been have been developed to date to overcome this problem (Mouritz and Cox, 2010). Examples are 3D woven or 3D braided preforms. Nevertheless, the production of such systems involves the adoption of dedicated equipment, which is often bulky, expensive and with limited versatility. Another option to generate a three-dimensional fibre pattern is to insert a reinforcing thread through the thickness of a standard two-dimensional preform, prior to resin infusion. 
These techniques are often referred to as $\mathrm{Z}$ reinforcing methods or through-the-thickness reinforcing (TTR) technologies. Stitching the layers of dry fabric together by interlocking one or more threads with two needles is a typical example of TTR technology (Mouritz and Cox, 2000), which, however, is only applicable to those setups where the backside of the preform is easily accessible. Even in those cases in which the arrangement of the needles is designed so that the thread (or threads) can be inserted from the same side of the preform, some form of support from the underside of the preform is still needed to allow enough room for the needles to operate.

Tufting represents one example of TTR methods for long fibre reinforced polymer composites which is intended to be used within the liquid resin injection manufacturing route, and involves inserting one single needle from one side only of the dry fibre preform, through its thickness (Cartie et al., 2006; Dell'Anno, 2007). The needle is then removed along the same direction, leaving a loop of reinforcing thread on the back side of the preform (figure 4a). The tuft loop is generated within a supporting material, typically a thermoplastic foam ${ }^{5}$, which can either be removed before infusion or left to become integral part of the structure. In the former case, a layer of nylon film is used to facilitate foam removal. The illustration in figure $4 \mathrm{~b}$ shows the thread arrangement in the basic form of tufting, where the foam is removed and the loop embedded in the matrix resin (right), or in the case in which the preform is penetrated only in part by the needle and hence partially reinforced (left). In the dry state, the reinforcing thread is held in place only by friction with the tuft not interlocked to any other thread. This gives tufting the added advantage over standard stitching of providing a virtually stress free preform with less crimp induced in the fibres.

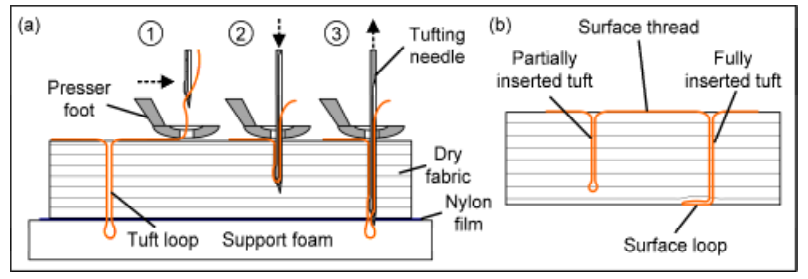

Fig. 4. Schematic of the thread insertion and arrangement in a tufted preform (Treiber, 2011).

Commercially manufactured tufting tools are now available as off-the-shelf products, ready to be interfaced with automated gantries or robotic arms ${ }^{6}$. The unit used for this study consists of a KSL KL150 tufting head mounted on a Kawasaki FS20N six-axis robot. The necessary control of the tufting process is achieved through a dedicated machine language and software, which are intended for use with Kawasaki robots. The complete set up is shown in figure 5; this unit has an adjustable speed and can insert up to 500 tufts per minute.
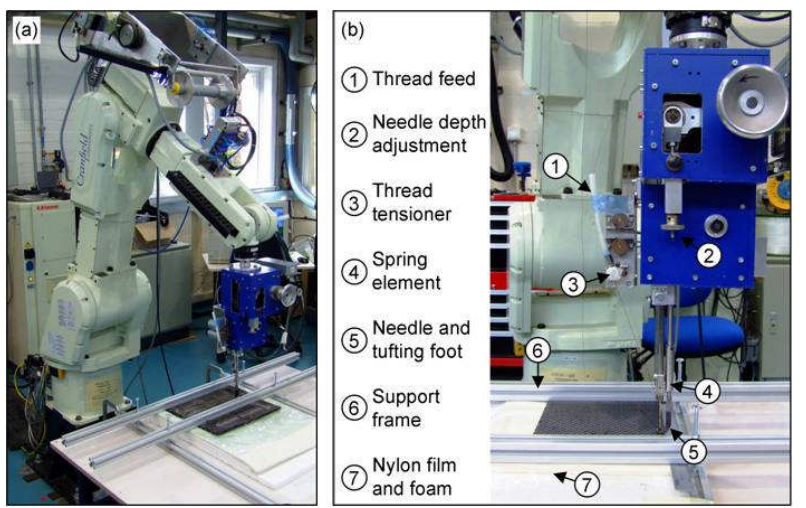

Fig. 5. (a) Kavasaki robot in operation and (b) detail of the KSL KL150 tufting head (Treiber, 2011). 
The structure and internal mechanisms of the tufting tool have to be robust enough to withstand the repeated loads deriving from the needle penetrating the preform, the tuftability of which depends not only on its thickness but also on the nature of the fabric. The technology has been designed for use with dry, unbindered preforms as a certain degree of motility is required to the fibres to allow needle and thread insertion. In fact, loose fabrics are easier to tuft and result in less stress for the unit. Processing of consolidated preforms or prepregs, in general, is not recommended and it could result in failure of the needle or damage to the unit; therefore, compatibility between tufting and ADFP technology, which produces consolidated, bindered preforms, is not obvious and needs to be verified, as further detailed in Section 3.1.

The needle design represents a very important aspect as it has to provide robustness with minimal cross section in order to reduce the extent of damage caused to the fibres. A newly designed needle has been developed recently by KSL: it is coded EP12 Nm230, it is $2.3 \mathrm{~mm}$ thick and wide and has a rounder and smoother shape than its predecessors. The diameter of the needle eye is $0.68 \mathrm{~mm}$ which necessarily limits the size of the threads usable for tufting. Apart from this limitation, a suitable thread must obviously be able to withstand being tufted without breaking. High twist counts usually confer the required flexibility in bending to the thread. To date, various threads have been identified as suitable for mechanical reinforcement applications and they are available in a variety of materials, as listed in table III (Treiber, 2011).

Table III. Examples of commercial threads suitable for tufting.

\begin{tabular}{llll}
\hline Thread material & Carbon & Glass & Kevlar \\
\hline Designation & Tenax ${ }^{\circledR}$ Carbon & EC9 68x3 S260 & Kevlar $^{\circledR}$ Tkt $30^{-}$ \\
Manufacturer & Schappe & Saint Gobain & Somac \\
Linear weight $[\mathrm{g} / \mathrm{km}]$ & 140 & 204 & 92 \\
Filament count & $2 \times 1000$ & $3 \times 411$ & $4 \times 134$ \\
Fibre density $\left[\mathrm{g} / \mathrm{cm}^{3}\right]$ & 1.76 & 2.60 & 1.44 \\
Dry cross section $\left[\mathrm{mm}^{2}\right]$ & 0.077 & 0.078 & 0.065 \\
Maximum load $[\mathrm{N}]$ & 144 & 103 & 132 \\
Tensile modulus $[\mathrm{GPa}]$ & 195 & 53 & 53 \\
Tensile strength $[\mathrm{MPa}]$ & 1848 & 1319 & 2018 \\
Ultimate strain $[\%]$ & 0.9 & 2.1 & 2.9 \\
\hline
\end{tabular}

Studies conducted to date on tufted composite laminates have demonstrated that this technology can potentially provide considerable improvement in delamination resistance with limited reduction of the in-plane properties. The Mode I interlaminar toughness of a carbon fibre/epoxy composite can be increased severalfold with a resulting reduction in tensile strength of approximately $10 \%$ and no measurable change in modulus (Dell'Anno et al., 2007). The apparent delamination toughness under Mode II configuration can be increased by up to $61 \%$ by inserting $0.5 \%$ areal density of carbon tufts in a non-crimp fabric preform (Treiber, 2011).

These figures strictly depend on the type of fabric tufted, in fact the level of modulus reduction is linked to the fibre misalignment induced by tufting, the extent of which is related to the inherent waviness of the pristine fabric itself. Improvements in delamination resistance often translate in enhanced damage tolerance. Compression after impact (CAI) tests performed on coupons tufted with glass or carbon demonstrated that the CAI strength of a carbon/epoxy laminate, after an impact of 15 Joules, is raised by $25 \%$ and $27 \%$, respectively, compared to the unreinforced composite (Dell'Anno et al., 2007).

In principle, the insertion of the thread can be perpendicular to the surface or at an angle (Dell'Anno, 2007), flat components or complex shapes can be processed (figure 6), and the distribution of insertions can be optimised according to the predicted loading pattern of the structure. Such a level of optimisation is best achieved via process automation. Tufting is ideally suited to work within a fully automated setup and, as previously explained, lends itself to be used in conjunction with the ADFP systems described in Section 2. Details on a compatibility test between these technologies are given in Section 3.1. 


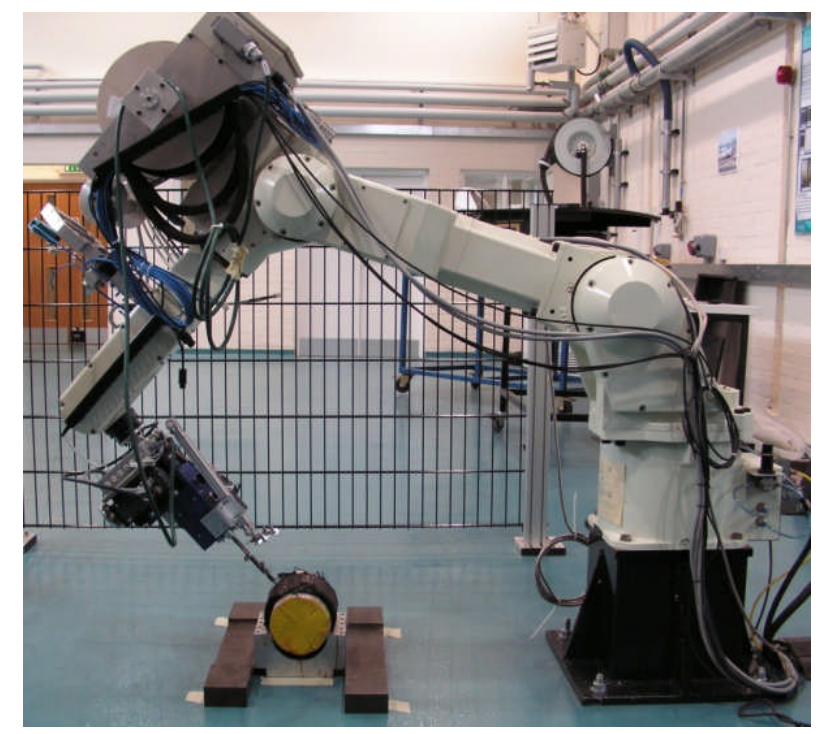

Fig. 6. Kawasaki FS $20 \mathrm{~N}$ robot unit with KSL KL 150 tufting head. In the picture the robot is tufting a preform with single curvature surface (Dell'Anno, 2007).

The efficiency and versatility of tufting as a TTR method can be exploited further when the technology is used as an alternative to mechanical fastening for joining subcomponents into complex structures (Mills, 2011). It is not the first time that a TTR technology is used as a fasteners replacement. Z-pins were studied at first for their potential in improving substantially the interlaminar strength and damage resistance of prepregged composites (Partridge and Cartié, 2005) but they currently find applications also as a cost effective part-joining system in the aerospace field. The use of $5 \mathrm{~m}^{2}$ of Z-pins for replacing 4600 titanium fasteners on the Northrop F18E/F military aircraft saved $17 \mathrm{~kg}$ on the final aircraft weight and approximately US\$ 83000 per aircraft (Hall, 2002).

Within the ADVITAC project, tufting is being used as a system to reinforce locally portions of the tail cone composite structure that are likely to be subjected to out-of-plane or shear loads or that might benefit from an improved damage tolerance. The use of tufting as a replacement for mechanical fastening between the tail cone skin and the reinforcing top-hat stringers is also being investigated.

\subsection{Tuftability tests on ADFP preforms}

Previous experience identifies the aramid thread as the most robust option among those detailed in table I: this thread is able to withstand the typical repeated load due to its insertion through the preform with a limited number of breakages. Nevertheless, the suitability of one or more threads depends strictly on the peculiar characteristics of the preform to be reinforced, therefore all grades were initially tested on two lab-scale ADFP preforms. A unidirectional and a $\left[0^{\circ} / 90^{\circ}\right]_{\mathrm{s}}$ panel, both consisting of 16 layers of Hexcel unidirectional, $6.35 \mathrm{~mm}$ wide carbon fibre tape (AS7 J12K with thermoplastic V800 veil) were laid-up with the unit described in Section 2 , equipped with a laser heating system. The consolidated preforms were $4.85 \mathrm{~mm}$ thick and were processed with the tufting tool described in Section 3.

The unidirectional preform passed the tuftability trials with no particular difficulties whereas the $\left[0^{\circ} / 90^{\circ}\right]_{\mathrm{s}}$ lay-up was more difficult to penetrate, with the tufting unit often stopped by overloading of the needle controlling motors. This is believed to depend on the fact that consolidated multiaxial lay-ups can limit severely the in-plane fibre movement, making it difficult for the needle to open up a path for the thread through the stack of plies. 
When unidirectional preforms were tested, the aramid product was the only grade of thread able to survive the initial feasibility trial without any breakage (see figure 7). The glass thread broke several times while the carbon thread did not survive a single insertion attempt. Although, based on the outcome of these initial trials, a potential fibre/thread combination is identified for the production of ADFP preforms reinforced by tufting, their mechanical performance remains to be established.

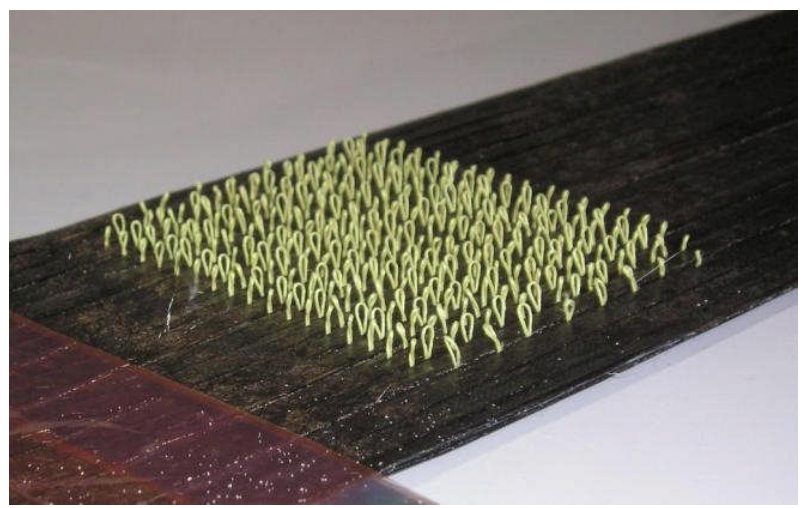

Fig. 7. Underside of the tufted ADFP unidirectional preform showing aramid thread loops of consistent length.

\section{Resin Infusion and Cure Monitoring using Optical Fibre Sensors}

A unique characteristic of optical fibre sensors is that they can be embedded within a composite part to monitor the manufacturing process because of their small diameter, typically 80-125 $\mu \mathrm{m}$ (Zhou and Sim, 2002). This offers unrivalled capability to monitor composite material processing on-line and in-situ. Once the fibres have been carefully laid-up by the ADFP system and reinforced locally by tufting, it is also important to monitor strictly the following manufacturing stages, in order to guarantee consistent quality in the final component. The progress of infusion, the degree of cure (extent of chemical reaction), and the strain are some of the important parameters that can be used to characterise the quality of the composite part during the production process. This section provides preliminary results on the monitoring of infusion and cure processes of a resin system using a fibre optic Fresnel refractometer (Cusano et al., 2001). The preforms utilised in this study were all prepared by Coriolis Composites using their ADFP unit, in order to test the suitability of this technique to be incorporated into a single manufacturing process in conjunction with dry fibre placement and tufting.

The refractive index of a material is determined by the types of molecular bonds it contains and by its density. The curing of a resin involves the conversion of the matrix resin from a low-molecular-weight viscous liquid or semi-solid to a highly cross-linked rigid structure. It has been shown that there is a correlation between the refractive index change and the degree of cure (Vacher et al., 2004; Dimopoulos et al., 2009) and that the refractive index of the resin is a nonlinear function of the temperature and the degree of cure. A photograph of a Fresnel refractometer sensor and the sensing element are shown in figure 8 and 9 , respectively. 


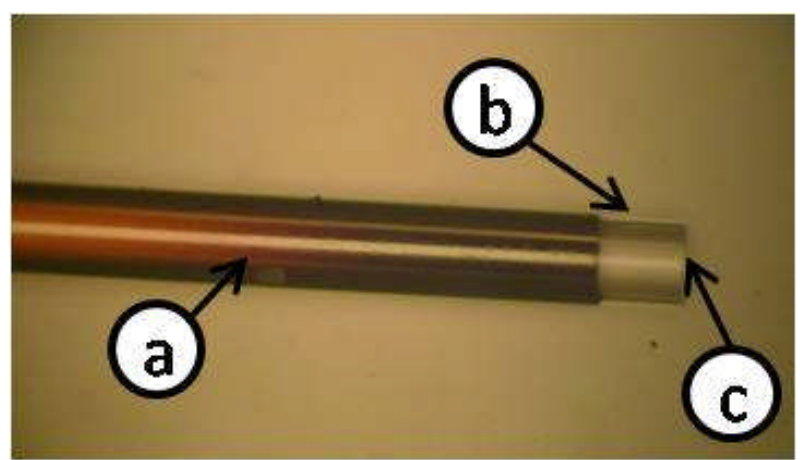

Fig. 8. Fresnel sensor, image of a cleaved end of the fibre: (a) polyimide coating, (b) fibre without coating, and (c) cleaved end face.

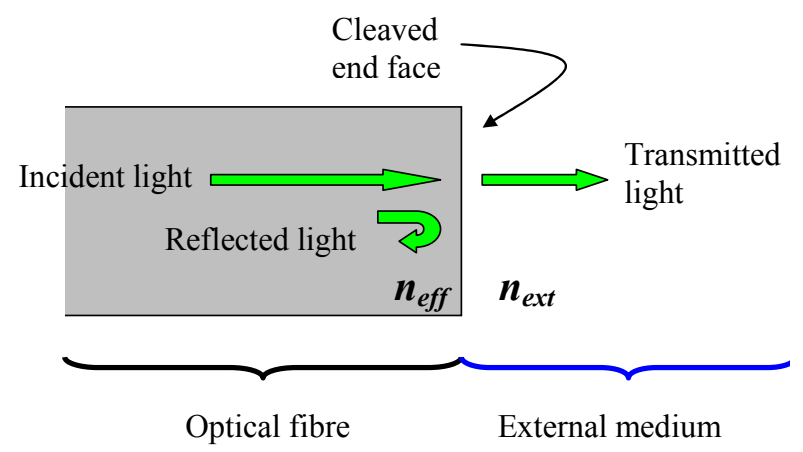

Fig. 9. Principles of the Fresnel refractometry. The symbol, neff represents the effective refractive index of the optical fibre, while next denotes the refractive index of the medium external to the fibre. The diameter of the optical fibre, with and without the polyimide coating, is 135 and 125 microns, respectively.

Light propagating in the core of the optical fibre is both partially transmitted to the external medium and partially reflected back into the fibre at the interface between the optical fibre and the external medium (figure 9). The amount of reflected light depends on the contrast in the refractive index between the two media. When the light reflected back into the fibre from the external medium is referenced to the light from the same source but reflected from a cleaved fibre end in air, the refractive index of the external medium can be determined. The reference is required to account for changes in source intensity and down lead losses. The refractive index $n$, measured by the Fresnel refractometry is governed by Equation (1) (Dimopoulos et al., 2009)

$$
n=n_{\text {eff }} \cdot \frac{1-\frac{\Delta}{\sqrt{R}}}{1+\frac{\Delta}{\sqrt{R}}},
$$

where

$$
\Delta=\frac{n_{e f f}-n_{a}}{n_{e f f}+n_{a}}
$$

and

$$
R=\frac{V_{a i r}}{V_{e x t}} .
$$

In Equation (1), $n_{\text {eff }}$ is the effective refractive index of the fibre mode, $n_{a}$ is the refractive index of air, $V_{\text {air }}$ is the voltage produced by the photo-detector that monitors the reflection from the fibre/air interface (i.e. the 
reference) and $V_{\text {ext }}$ is the voltage produced by the photo-detector that measures the reflection from the fibre/external-medium interface.

Figure 10 is a schematic diagram of a Fresnel refractometer which is configured to measure the refractive index of a sample at two locations. The reference channel records the light intensity reflected back from the fibre/air interface which is used to normalise the signals obtained from the two measurement channels.

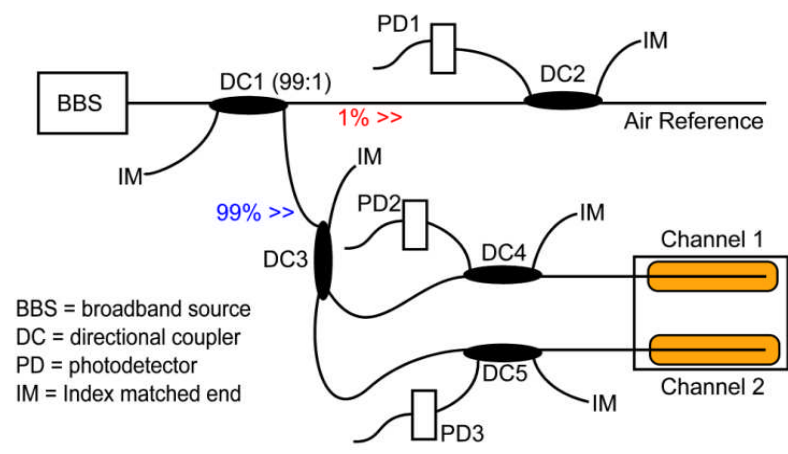

Fig. 10. Schematic diagram of a 2-channel Fresnel refractometer.

When monitoring resin infusion, the reflected signal decreases dramatically when resin covers the end face of the fibre because of the difference in refractive index between air and resin. This signal attenuation is used to indicate resin arrival at the location of the sensor during the infusion process. Subsequently, as the resin cures the reflected signal will change as the refractive index of the resin changes, which can be exploited as a phenomenological measurement of the extent of the cure process or degree of cure.

Two Fresnel sensors were surface mounted onto a dry preform measuring $620 \mathrm{~mm} \times 720 \mathrm{~mm}$, one at the bottom and the other at the top surface. The sample was placed in a vacuum bag and placed in an oven. An HexFlow RTM6 resin which was maintained at a temperature of $85^{\circ} \mathrm{C}$ was infused through the thickness, from the bottom to the top of the sample. Data logging of the Fresnel sensor system was started after the vacuum was established and about 3.2 minutes before infusion was started (figure 11-left). The sample was left to cure at an isotherm of $180^{\circ} \mathrm{C}$ for 4 hours.
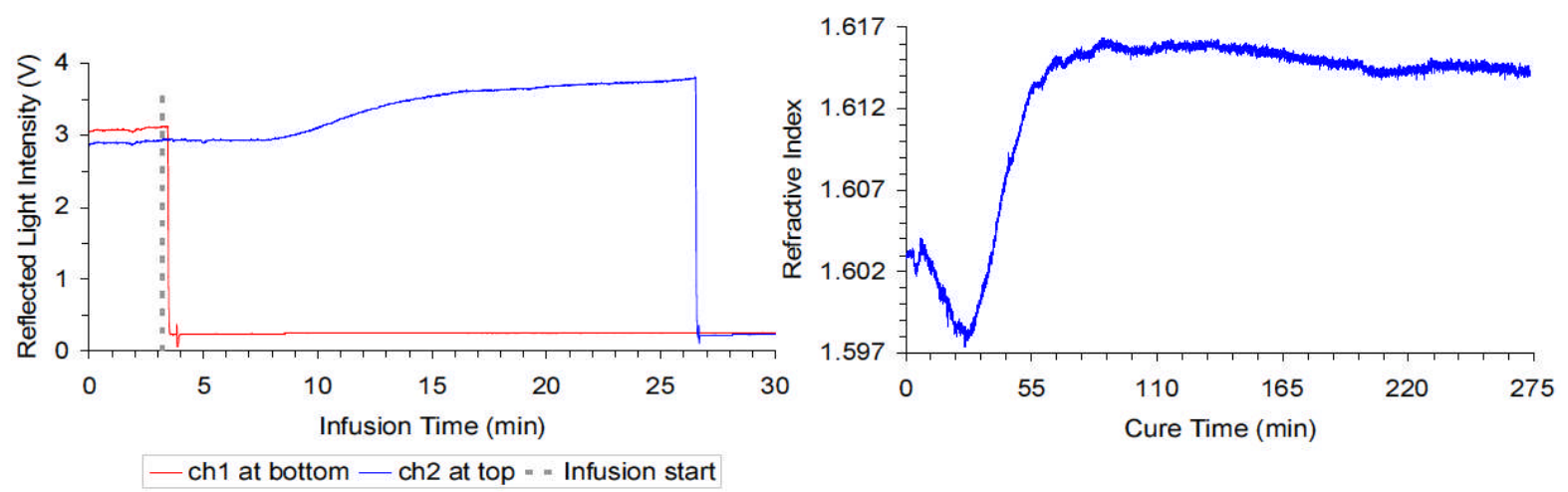

Fig. 11. (Left) Signals from the two channels of a Fresnel infusion sensor obtained during the infusion of RTM6 resin. Channel 1 (red) and channel 2 (blue) located at the bottom and top of the preform, respectively. The dashed line indicates the time at which infusion was started.

(Right) Evolution of the refractive index of the resin recorded by channel 2 during the cure of RTM6 resin.

Channel 1, located at the bottom of the sample, detected the arrival of resin 15 seconds after the start of infusion and 1384 seconds (or 23 minutes) later the resin was detected by channel 2 at the top of the preform. 
These times are indicated in figure 11-left by the sudden attenuations in the 2 signals. Figure 11-right shows the evolution of the refractive index of the resin during cure measured by channel 2 . The decrease in refractive index during the first 25 minutes signifies the rise in temperature from $85{ }^{\circ} \mathrm{C}$ to $180{ }^{\circ} \mathrm{C}$. The rapid increase in the refractive index up to about 90 minutes of cure time denotes the bulk of the cure reaction. Thereafter the refractive index is much stable thus indicating that the resin is approaching full cure.

In a separate experiment, two Fresnel sensors were placed on top of a dry preform measuring $450 \mathrm{~mm} \mathrm{x}$ $450 \mathrm{~mm}$, one near the edge $(40 \mathrm{~mm})$ and the other in the centre. The sample was infused with Cycom RTM890 resin which was maintained at a temperature of $90^{\circ} \mathrm{C}$ and was subsequently cured at an isotherm of $180^{\circ} \mathrm{C}$ over 3 hours. The infusion and cure processes are similar to those described above. Data logging of the Fresnel sensor was started about 4 minutes before infusion was started (figure 12-left).
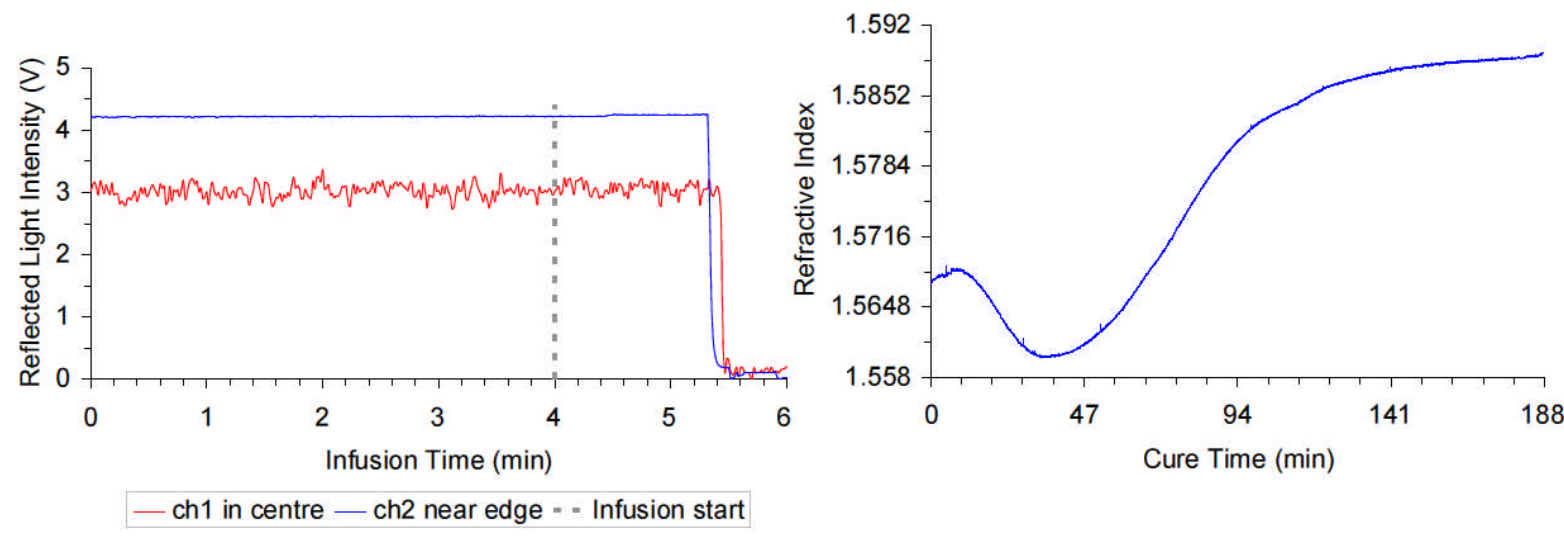

Fig. 12. (Left) Signals from the two channels of a Fresnel infusion sensor obtained during the infusion of RTM890 resin. Channel 1 (red) located at centre and channel 2(blue) located at the edge $(40 \mathrm{~mm})$, both on top of the preform. The dashed line indicates the time at which infusion was started. (Right) Evolution of the refractive index of the resin recorded by channel 2 during the cure of RTM890 resin.

Channel 2, located near the edge $(40 \mathrm{~mm})$ of the sample, detected the arrival of resin 1.5 minutes after the start of infusion while channel 1, which was in the centre of the sample, detected the arrival of resin approximately 6 seconds later. The onset of the sudden attenuation in the signals of figure 12-left marks the resin arrival at the sensor locations. Figure 12-right shows the evolution of the refractive index of the resin during cure measured by channel 2 near the edge of the sample. The first 38 minutes during which the refractive index decreases represent the temperature rise from $90{ }^{\circ} \mathrm{C}$ to $180^{\circ} \mathrm{C}$. Most of the cure reaction takes place from approximately 38 to 140 minutes during which the refractive index increases at a fast rate. Thereafter the refractive index becomes constant which indicates that the resin is approaching full cure.

To investigate the resin infusion and cure within a sample, two Fresnel sensors were embedded within the middle layer of an 8 layer dry preform that was hand laid. The sample measured $400 \mathrm{~mm} \times 400 \mathrm{~mm}$. One sensor was $40 \mathrm{~mm}$ from the edge while the other was in the centre but on the same layer of the preform. The sample was subsequently infused with Prism EP2400 resin which was maintained at a temperature of $90{ }^{\circ} \mathrm{C}$ before being cured at an isotherm of $180^{\circ} \mathrm{C}$ for approximately 4 hours.

Data logging of the Fresnel sensor was started 16.45 minutes before the infusion was started. Channel 2, located near the edge $(40 \mathrm{~mm})$ of the sample, detected the arrival of resin 15 minutes after the start of infusion. Channel 1, which was situated in the centre of the sample, detected the arrival of resin approximately 15 seconds later. The sudden attenuation in the signals of figure 13-left marks the resin arrival at the sensor locations. Figure 13-right shows the evolution of the refractive index of the resin during cure measured by channel 2. Similarly, the temperature rise to an isotherm of $180^{\circ} \mathrm{C}$ is signified by the first 50 minutes during which the refractive index is predominantly decreasing. The bulk of the chemical cure reaction takes place 
between 50 and 130 minutes. Thereafter the refractive index is approximately constant meaning that the resin is almost fully cured.
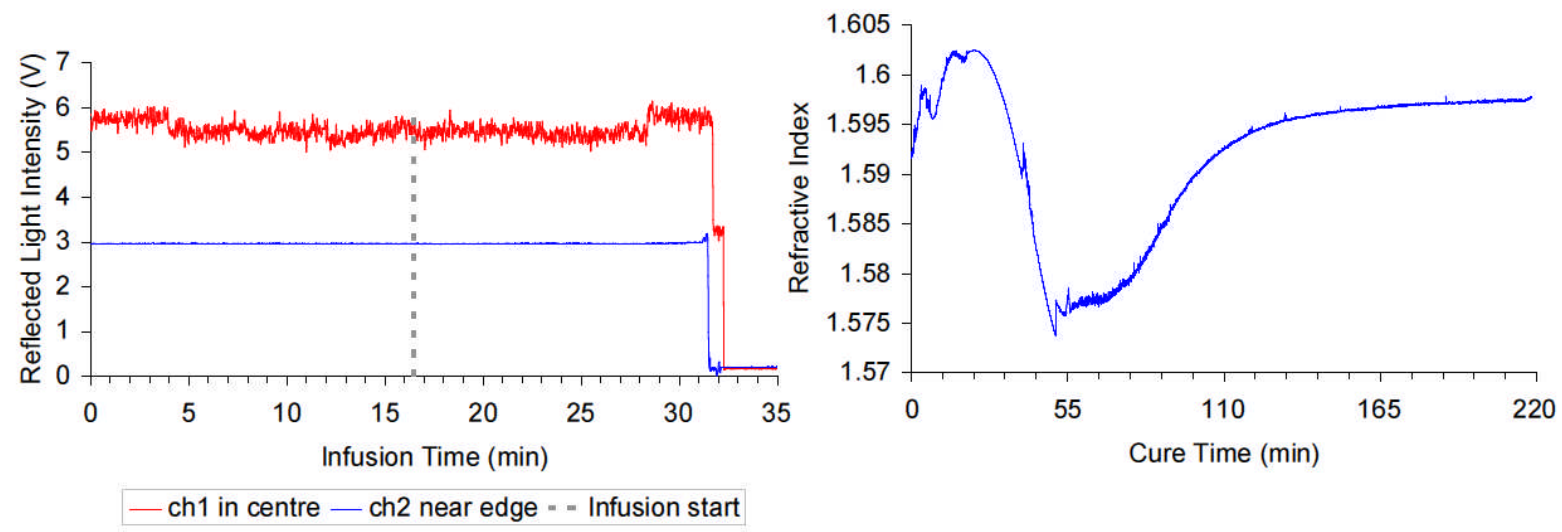

Fig. 13. (Left) Signals from the two channels of a Fresnel infusion sensor obtained during the infusion of EP2400 resin. Channel 1(red) located at centre and channel 2(blue) located at the edge $(4 \mathrm{~cm})$, both inside the preform. The dashed line indicates the time at which infusion was started. (Right) Evolution of the refractive index of the resin recorded by channel 2 during the cure of EP2400 resin.

The preliminary experiments reported here have demonstrated the capability to monitor resin infusion and subsequently to measure the evolution of the refractive index of a resin using a fibre optic Fresnel refractometer during industrial composite material processing. To determine the degree of cure of the resin via this refractive index change, a calibration of the Fresnel sensor is required. Further experiments will be performed to characterise the resin cure using differential scanning calorimetry (DSC) measurements in order to determine the correlation between the degree of cure and refractive index of the resin (Dimopoulos et al., 2009).

\section{Conclusions}

The combination of technologies described in this paper appears well suited to operate within a single, innovative and cost-effective manufacturing process, for the production of lighter, functional and damage tolerant composite structures.

Automated dry fibre tow placement technology has the potential of exploiting the full capability of the fibres by tailoring the fibre architecture to the specific needs of the component, while still retaining the economic benefits of liquid moulding processes. The use of a laser heating system on the ADFP unit has demonstrated that dry stabilised tapes can be laid-up to the required ply sequence obtaining quick consolidation of the preform. Once impregnated with standard epoxy resins, the cured laminates exhibit promising mechanical performance, in line with expectations.

Tufting can contribute to the reduction of structural redundancy by locally reinforcing critical areas of the component while providing the possibility of replacing fully or at least in part the need for mechanical fastening. The possibility of tufting consolidated preforms with fibrous threads was investigated; aramid tufts were successfully inserted while glass and carbon options had to be discarded for their incompatibility with the ADFP preform.

Optical fibre sensors have proven their effectiveness in on-line monitoring resin infusion and curing and can now be integrated into fully automated processes, which offer enough accuracy to place the sensors within the structure with an adequate level of precision. 


\section{References}

Cartié D.D.R. and Dell'Anno G., Poulin E., Partridge I.K. (2006). 3D Reinforcement of stiffener-to-skin T-joints by Z-pinning and tufting. Engineering Fracture Mechanics, 73: 2532-2540.

Cusano A. and Breglio G., Giordano M., et al. (2001). Optoelectronic characterization of the curing process of thermosetbased composites. J. Opt. A-Pure And Applied Optics, 3: 126-130.

Dell'Anno G. (2007). Effect of tufting on the mechanical behaviour of carbon fabric/epoxy composites (PhD thesis), Cranfield University, Cranfield.

Dell'Anno G. and Cartié D.D.R., Partridge I.K., Rezai A. (2007). Exploring mechanical property balance in tufted carbon/fabric composites. Composites: Part A, 38: 2366-2373.

Dimopoulos A. and Buggy S.J., Skordos A.A., James S.W., Tatam R.P., Partridge I.K. (2009). Monitoring cure in epoxies containing carbon nanotubes using an optical fibre Fresnel refractometer. J. Applied Polymer Science, 113: 730-735.

Grant C.G. (2000). Fibre placement process utilization within the worldwide aerospace industry. Proceedings of SAMPE 2000, (May 2000, Long Beach, CA).

Hall T. (2002). Automation of Z-fibre for complex shapes. Available at cmtc.scra.org/pubs/fall_2002.pdf (accessed September 2010).

Izco L. and Isturiz J., Motilva M. (2006). High Speed Tow Placement System for Complex Surfaces with Cut/Clamp/\& Restart Capabilities at $85 \mathrm{~m} / \mathrm{min}$ (3350 IPM). Aerospace Manufacturing and Automated Fastening Conference and Exhibition, (September 2006, Toulouse, France).

Koh, T.M. and Feih S., Mouritz A.P. (2011). Experimental determination of the structural properties and strengthening mechanisms of z-pinned composite T-joints. Composite Structures, 93: 2222-2230.

Mills A.R. (2011). Flying high with carbon fibre. Materials World, May 2011.

Mouritz A.P. and Cox B.N. (2000). A mechanistic approach to the properties of stitched laminates. Composites: Part A, 31: 1-27.

Mouritz A.P. and Cox B.N. (2010). A mechanistic interpretation of the comparative in-plane mechanical properties of 3D woven, stitched and pinned composites. Composites: Part A, 41: 709-728.

Partridge I.K. and Cartié D.D.R. (2005). Delamination resistant laminates by Z-Fiber ${ }^{\circledR}$ pinning. Part I manufacture and fracture performance. Composites: Part A, 36: 55-64.

Ridgard C. (2009). Out of autoclave composite technology for aerospace, defense and space structures. Proceedings of SAMPE 2009, (May 2009, Baltimore, MD).

Sloan J. (2008). ATL and AFP: Signs of evolution in machine process control. High-Performance Composites, September 2008. Available at www.compositesworld.com/articles/atl-and-afp-signs-of-evolution-in-machine-process-control (accessed September 2010).

Treiber J.W.G. (2011). Performance of tufted carbon fibre/epoxy composites (PhD Thesis). Cranfield University, Cranfield. Vacher S. and Molimard J., Gagnaire H., et al. (2004). A Fresnel's reflection optical fibre sensor for thermoset polymer cure monitoring. Polymers \& Polymer Composites, 12: 269-276.

Zhou G. and Sim L.M. (2002). Damage detection and assessment in fibre-reinforced composite structures with embedded fibre optic sensors - review. Smart Mater. Struct. 11: 925-939.

\footnotetext{
1 ADVITAC (Advanced Integrated Composite Tail Cone) website. Available at www.advitac.eu (accessed June 2011).

2 Thermoset prepreg slit tapes, thermoplastic prepreg slit tapes and stabilized dry tows.

3 ABB Robotics website. Available at www.abb.com/robots (accessed June 2011).

4 KUKA Robot Group website. Available at www.kuka-robotics.com (accessed June 2011).

5 A typical example is Airex ${ }^{\circledR}$ R63 closed cell PVC foam.

${ }^{6}$ Keilmann Group website. Available at www.ksl-lorsch.de (accessed June 2011).
} 